\title{
OBSTACLE DETECTION SYSTEM INVOLVING FUSION OF MULTIPLE SENSOR TECHNOLOGIES
}

\author{
C. Gianni ${ }^{\mathrm{a}}$, M. Balsi ${ }^{\mathrm{a}}$, S. Esposito ${ }^{\mathrm{b}}$, P. Fallavollita ${ }^{\mathrm{a}, \mathrm{b}}$ \\ ${ }^{a}$ DIET, Dept. of Information Engineering, Electronics and Communications, "La Sapienza" University of Rome, Italy - \\ (carmine.gianni, marco.balsi, paolo.fallavollita)@uniroma1.it \\ boben s.r.1., Sassari, Italy - esposito@oben.it
}

KEY WORDS: Obstacle avoidance, UAV, Data fusion

\begin{abstract}
:
Obstacle detection is a fundamental task for Unmanned Aerial Vehicles (UAV) as a part of a Sense and Avoid system. In this study, we present a method of multi-sensor obstacle detection that demonstrated good results on different kind of obstacles. This method can be implemented on low-cost platforms involving a DSP or small FPGA. In this paper, we also present a study on the typical targets that can be tough to detect because of their characteristics of reflectivity, form factor, heterogeneity and show how data fusion can often overcome the limitations of each technology.
\end{abstract}

\section{INTRODUCTION}

Today several UAVs implement obstacle detection, but often they rely on Stereo-cameras, ultrasonic sensors or scanning LiDAR sensors. Every single technology poses a limitation since obstacles are very different each other in form factor, reflectivity, composition. Lightweight and low-cost UAVs, in particular, need obstacle avoidance feature, for safety, ease of operation, and in perspective for upcoming regulations.

\subsection{State of the art}

Since the introduction of the first UAVs equipped with ultrasonic sensors to detect obstacles a lot of progress has been done. Obstacle detection is a non-trivial task. Technologies for such purpose can be divided into active and passive ones. In the former, the system is based on a transmitter that irradiates the target and a receiver that gets back the signal coming from it, as is the case, e.g., for SONAR and RADAR. On the other hand, in passive technologies there is just a sensor that receives signals coming from environment. This kind of sensors includes in particular stereo-cameras or optical-flow cameras.

Most existing systems rely exclusively on visual servoing (cameras, stereo-cameras) and SLAM techniques (DJI, 2017; Kanellakis, 2017; Sabatini, 2013; Burri, 2015; Fallavollita, 2012). These systems are very good when operating in favorable light and environment conditions, but suffer with certain kind of obstacles like wires and nets, and operation in poor light conditions like night-time or foggy scenarios. Moreover, these systems (especially those using mapping) require a lot of computation power to react in real-time situations.

Fusion of heterogeneous sensors has been used to enhance reliability of obstacle detection. Scanning LiDARs and RADARs have been used in conjunction with visible and multispectral imaging sensors, as well as ultrasound (Nieuwenhuisen, 2013; Fasano, 2016; Holliger, 2015; Gageik, 2015). Fusion methods often involve Kalman filtering (Khalegi, 2013; Welch, 2006) but (Gageik, 2015) chose a weighted filter, based on a rule system, to reduce computational burden. The latter system is targeted to low-cost/low-weight UAV systems, while most others involve heavy and expensive sensor sets that are only justified for relatively large systems and most demanding tasks.

\section{CHOSEN TECHNOLOGIES}

In this work, our attention was focused on active technologies. Sensors have been chosen to be practical for use on board a light and low-cost UAV. The three technologies integrated in the multi-sensor system are characterized by being complementary w.r.t. nature of targets, to get the widest spectrum of detected obstacles.

\subsection{FMCW Radar}

An FMCW Radar is a kind of Radar that transmits a frequencymodulated microwave signal and can detect the distance and the speed of an obstacle by analyzing the frequency spectrum of the reflected signal.

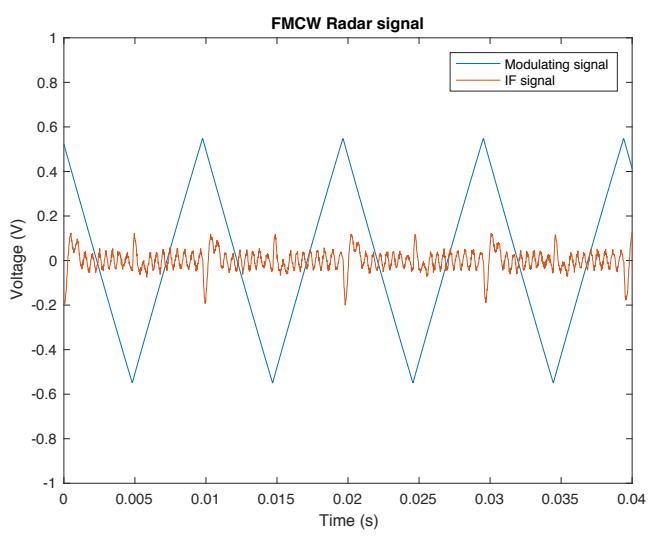

Figure 1. FMCW Radar Waveform

Figure 1 shows the modulating wave of a FMCW RADAR and the signal at the IF output; the typical modulation patterns are Sawtooth or Triangular, with the former used for range measurements and the latter for both range and velocity. The effectiveness of a RADAR is limited by several factors like the RCS (Radar Cross Section) of the target and the wavelength of the RADAR signal with respect to the dimension of the target. In our project, we chose an IVS-167 RADAR (Innosent, 2013). 


\subsection{LiDAR}

LiDAR sensors use a LASER source and a receiving photodiode to measure the distance of an obstacle. Due to the shorter wavelength of light, LiDARs can be a lot more accurate than RADARs in detecting obstacles: here the limitations are posed by the reflectivity of the target and by its form factor. The chosen sensor is LidarLite (PulsedLight3D, 2015), a low-cost laser range finder characterized by low weight and good ranging capabilities up to $30-40 \mathrm{~m}$ depending on obstacle reflectivity.

\subsection{SONAR}

SONAR uses sound waves reflection to detect a target. The sensor chosen (MaxBotix, 2015), uses ultrasonic waves in the range of $40 \mathrm{KHz}$ and can detect obstacles up to 7 meters away depending on their size. This sensor is characterized by a high FOV, greater than $90^{\circ}$ of aperture. SONARs are most efficient on hard surfaces, and have relatively wide main lobe. Normally, they are rather limited in range.

\section{METHOD}

The proposed method is based on fusing, by means of a Kalman filter, data coming from multiple sensors, each one characterized by a different technology. Here the challenge is that data coming from sensors are very heterogeneous, every sensor in fact has its own operating frequency, range and data format. Figure 2 shows the proposed system architecture. In the next subsections we'll describe the function of each block.

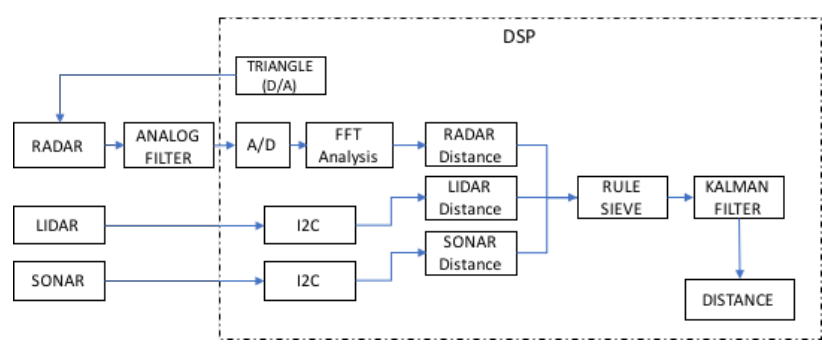

Figure 2. System Architecture

\subsection{Rule sieve}

Before putting raw data into a Kalman filter, the information must first be manipulated and suitably "sieved". A rule filter has been implemented which does a coarse selection of the data to be passed to the Kalman filter. Rules applied to data are the following:

1. Maximum and minimum Range constraints

2. Measurements sanity check

3.1.1 Maximum and minimum Range constraints: Every sensor has its own operating range, so data coming for example from ultrasound sensor near its full-scale range get dropped by the filter. In Table 1 the chosen range constraints are shown.

\begin{tabular}{|l|l|l|}
\hline & Min Range $(\mathrm{m})$ & Max Range $(\mathrm{m})$ \\
\hline RADAR & 2 & 40 \\
\hline LiDAR & 0.1 & 30 \\
\hline SONAR & 0.1 & 7 \\
\hline
\end{tabular}

Table 1. Range constraints
3.1.2 Measurements sanity check: Even if a measurement appears to be correct, it can be distorted by the limits of the technology of a sensor. For instance, LiDAR sensor measurements are usually very precise if compared to the ones of FMCW RADAR and SONAR, however they can be a lot less accurate than the other two because LiDAR sensors have very tight FOV, so they can miss obstacles that have narrow form factors like nets or wires.

The rule filter compares the measurements coming from the three sensors and if the other two sensors have taken a valid measurement and measure a distance shorter than that one of the LiDAR, the latter gets dropped. Dropping a measurement means to invalidate it by passing a 0 value into the $\mathrm{H}$ matrix of the Kalman filter for that measurement.

\subsection{Kalman Filter}

Kalman filters are often used in data fusion because of their ease of implementation, and optimality in a mean-squared error sense (Khalegi, 2013). To implement the Kalman filter we have used the well-known equations for the two-step discrete Kalman filter cycle (Welch, 2006)

Time update (prediction)

$$
\begin{gathered}
\hat{x}_{k}^{-}=A \hat{x}_{k-1}^{-}+B u_{k-1} \\
P_{k}^{-}=A P_{k-1}^{-} A^{T}+Q
\end{gathered}
$$

Measurement update (correction)

$$
\begin{gathered}
K_{k}=P_{k}^{-} H^{T}\left(H P_{k}^{-} H^{T}+R\right)^{-1} \\
\hat{x}_{k}=\hat{x}_{k}^{-}+K_{k}\left(z_{k}-H \hat{x}_{k}^{-}\right) \\
P_{k}=\left(I-K_{k} H\right) P_{k}^{-}
\end{gathered}
$$

Here the desired physical quantity in output from the filter is the distance of a target. Distance has been chosen as the state of the filter ( $\hat{x}_{k}$ is the distance estimation), and the three measurements $\left(z_{k}\right)$, coming from the sensors are fed into the filter in the measurement update phase, where the a posteriori state estimate is computed. $K_{k}$ is the Kalman filter gain. The remaining Kalman filter parameters are defined as follows:

$$
\begin{gathered}
A=1 \quad R=\left(\begin{array}{ccc}
r_{R} & 0 & 0 \\
0 & r_{L} & 0 \\
0 & 0 & r_{R S}
\end{array}\right) \\
B=0 \\
Q=10^{-5} \quad H=\left(\begin{array}{c}
h_{R k} \\
h_{L k} \\
h_{S k}
\end{array}\right)
\end{gathered}
$$

- $A$ is the state transition matrix, because it represents the evolution of the single state of the system (the distance).

- $B$, the control matrix is zero, because we assume not having any external input $\left(u_{\mathrm{k}}\right)$ to the system.

- $H$ matrix indicating the observation model, is a $3 \times 1$ matrix containing the three values $\left(h_{R k}, h_{L k}, h_{S k}\right)$ where the $\mathrm{R}, \mathrm{L}$ and $\mathrm{S}$ subscripts refer to RADAR, LiDAR and SONAR respectively. 
These values can be ones or zeros depending on whether the measure of each of the three sensors has passed or not the previous rule filter.

- $R$ is the measurement noise covariance and is populated with the noise of the three sensors $\left(r_{R}=0.36, r_{L}=0.0025, r_{S}=0.01\right)$ as it is obtained from technical specs of each sensor.

- $Q$ is the process noise covariance, its value is not so obvious and often must be guessed as a first assumption and then tuned by experimentation. Here we have chosen a low value of $Q$ assuming a low process noise.

The last parameter to define is $P_{0}$, the initial estimate error covariance. It is not critical, it just needs to be different from zero, so we set $P_{0}=1$.

\section{RESULTS}

In order to test the assumptions made so far, several tests have been carried out. The experiments have been done in our laboratories and in an outdoor environment and we analyzed various kind of obstacles known to pose problems in detection with current technologies, so we focused on poles, nets, vegetation, windows and persons.

\subsection{Experimental Setup}

The FMCW RADAR chosen for the experiment is characterized by a very directional pattern as shown in Figure 3, its output at the IF pin is in base band frequencies, but the output signal must be filtered and amplified to be used correctly. This is because the output signal is in the order of few $\mathrm{mV}$, and furthermore it presents spurious frequencies that feed into the output signal from the input modulating signal. To this purpose, we designed a filter-amplifier chain made of a stage of Sallen-Key filter and a two-stage amplifier. The difficulties that we encountered here were in limiting the noise of the circuit to get a good signal-tonoise ratio at the output, so we chose a low noise op-amp (MAX44252) and operated in dual supply to minimize the common-mode noise. In Figure 4 the response of the filter in magnitude and phase is shown.

The other two sensors, the LidarLite LASER rangefinder and the Ultrasonic (SONAR) sensor have been interfaced to the measuring system through their $\mathrm{I}^{2} \mathrm{C}$ serial interface.

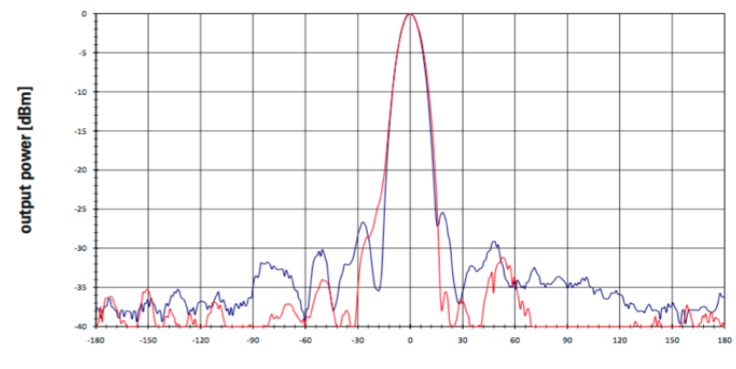

angle [०]

Figure 3. IVS-167 Antenna vertical (blue) and horizontal (red) pattern

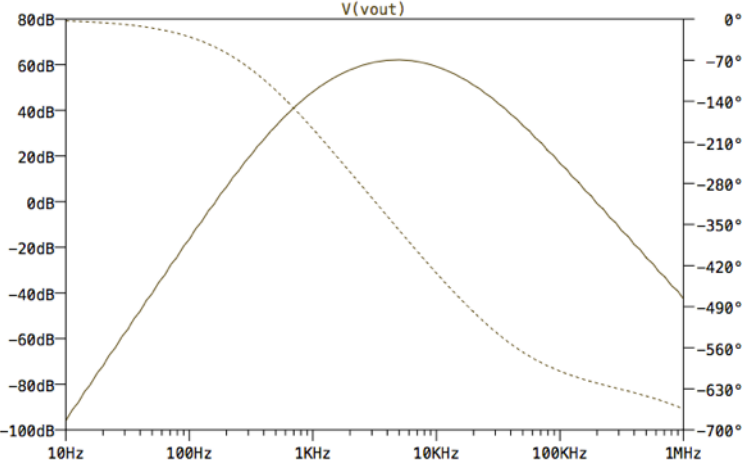

Figure 4. Response of the FMCW Radar Filter - magnitude (solid), phase (dotted)

In a first setup, depicted in Figure 5, just to validate the system, the output of the three sensors has been read by a PC running Matlab, implementing the decision filter based on sieve and Kalman filter.

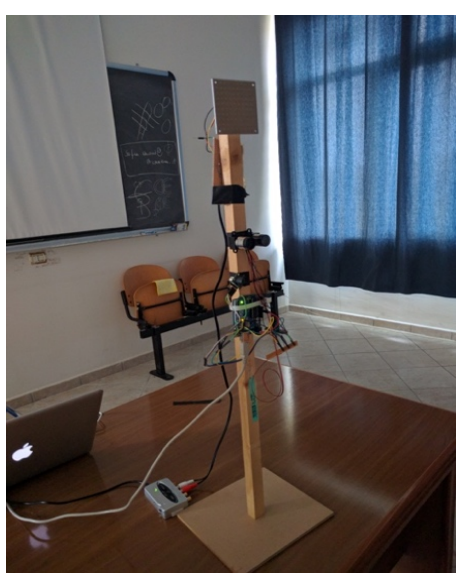

Figure 5. First Experimental setup

Once the system principle has been validated, to make the system portable and mountable on board a UAV, we made a second experimental setup. This time the core of the multi-sensor system is a DSP from ST Microelectronics, STM32F446RC. This unit offers a high performance-to-cost ratio. In Table 2 some key data from its datasheet are reported (ST, 2015):

\begin{tabular}{|l|l|}
\hline \multicolumn{2}{|l|}{ STM32F446RC } \\
\hline Architecture & ARM Cortex M4 32 bit + FPU \\
\hline Performance & $180 \mathrm{MHz}-225$ DMIPS \\
\hline Flash memory & $256 \mathrm{kB}$ \\
\hline RAM memory & $128 \mathrm{kB}$ \\
\hline
\end{tabular}

Table 2. STM32F446RC Specifications

Figure 6 and Figure 7 show the system integrated on a PCB and mounted on a plastic box. 


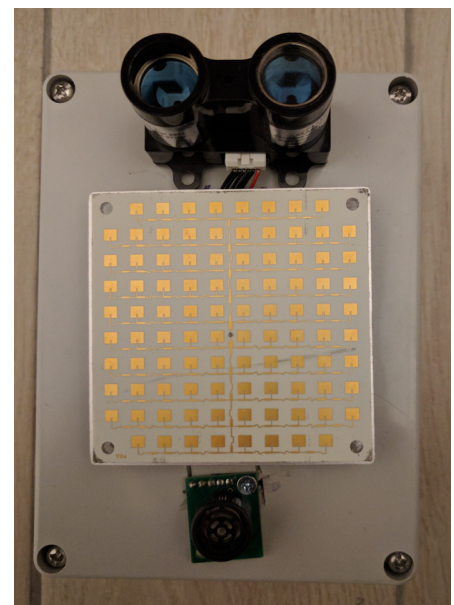

Figure 6. Second Experimental setup

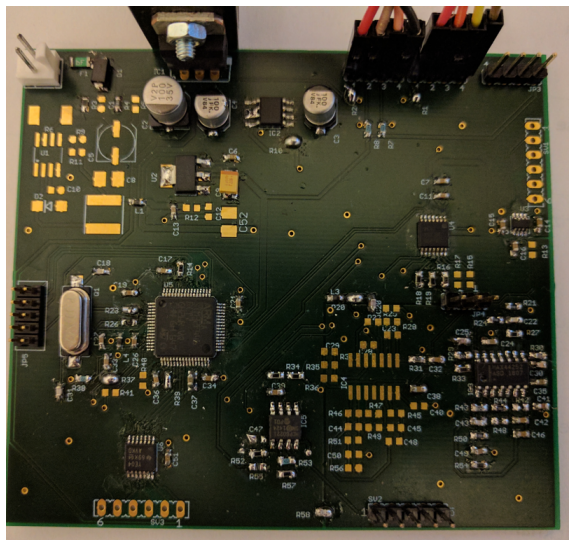

Figure 7 DSP Multi-sensor Board

\subsection{Case studies}

In the following subsections, the various case studies addressed in this work are shown. Measurements are made by pointing the device towards the target for 5 seconds with an output rate of 2 $\mathrm{Hz}$, thus acquiring all the sensors data, just for simplicity, at the rate of the slowest sensor (RADAR). Anyway in the future, since the Kalman Filter allows it, we will work at multiple rates.

4.2.1 Wall: As a first case study, here we propose a brick wall, clearly it's a simple obstacle, but we'll take this case as reference for the other tests. Figure 8 shows the output of the three sensors and in the line with circle markers shows the output from our Kalman filter.

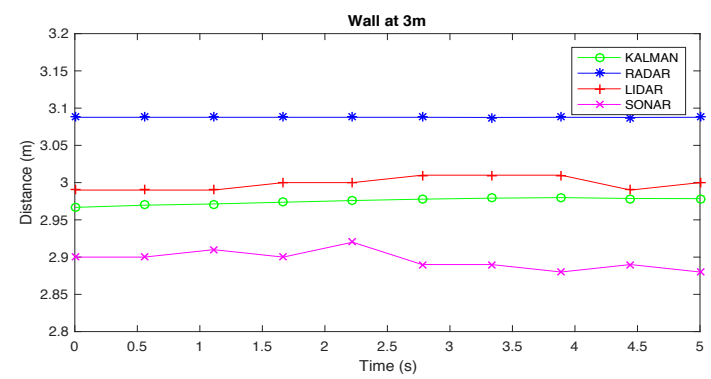

Figure 8 . Wall at $3 \mathrm{~m}$ distance
Figure 9 shows $\mathrm{K}$ gains of the filter (a) and $\mathrm{P}$, the a posteriori error covariance estimate. The trends of the curves are as expected from Kalman filter theory. $\mathrm{K}$ gain relative to the RADAR sensor is particularly low because the filter is not considering it, preferring the information coming from LiDAR and SONAR.

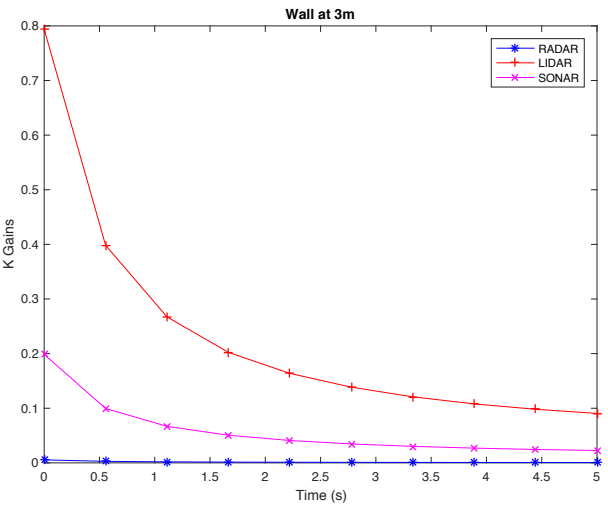

(a)

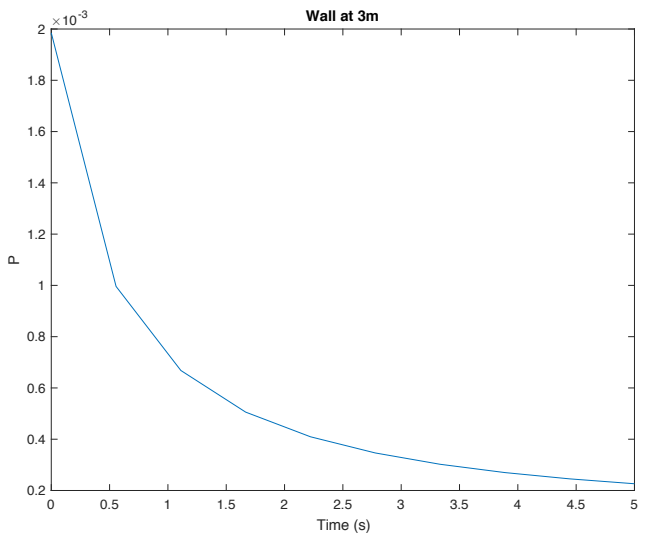

(b)

Figure 9 (a) K gains, (b) P error covariance

Figure 10 shows the results for the same wall at $10 \mathrm{~m}$ distance, this example shows how the Kalman output ties to the LiDAR output, since it is more confident of its measurements, while the rule sieve has discarded the SONAR measurement altogether.

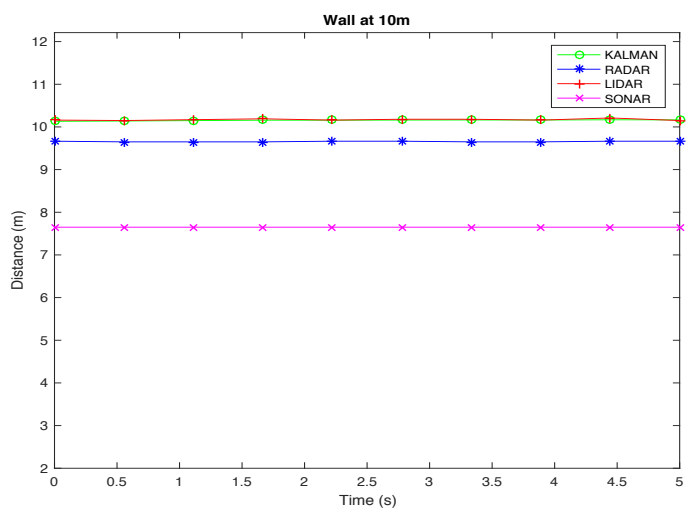

Figure 10. Wall at $10 \mathrm{~m}$ distance 
4.2.2 Window: The second case study is a window (Figure 11). This kind of obstacles stress the difficulties of the LiDAR technology that is deceived by the glass.

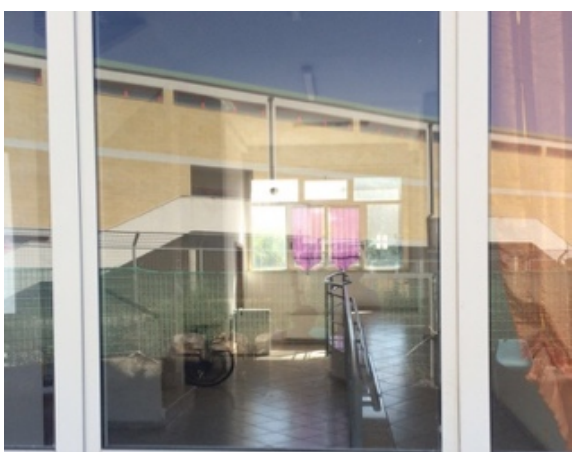

Figure 11. Window used for the experiment

This is evident in Figure 12: in fact, the LiDAR data is totally wrong most of the time. Nevertheless, the Kalman filter output is still good, because the rule filter discarded the measurements of the LiDAR.

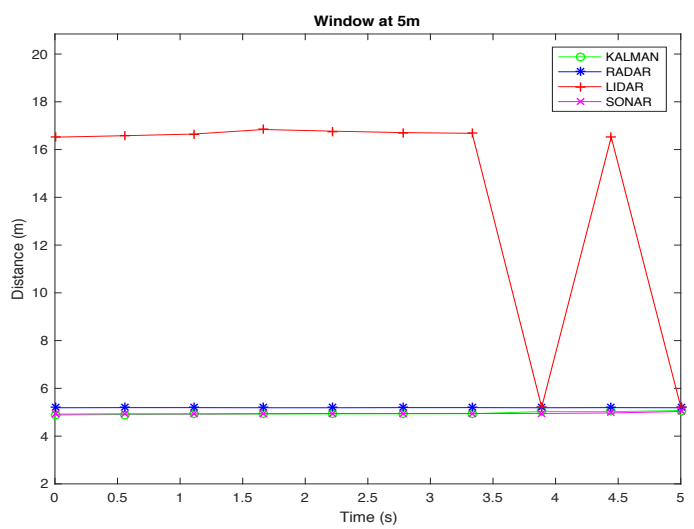

Figure 12. Window at $5 \mathrm{~m}$ distance

4.2.3 Pole: A difficult obstacle is a pole (Figure 13), due to its long and thin form factor it is hard to detect at distance with sensors characterized by a narrow FOV.

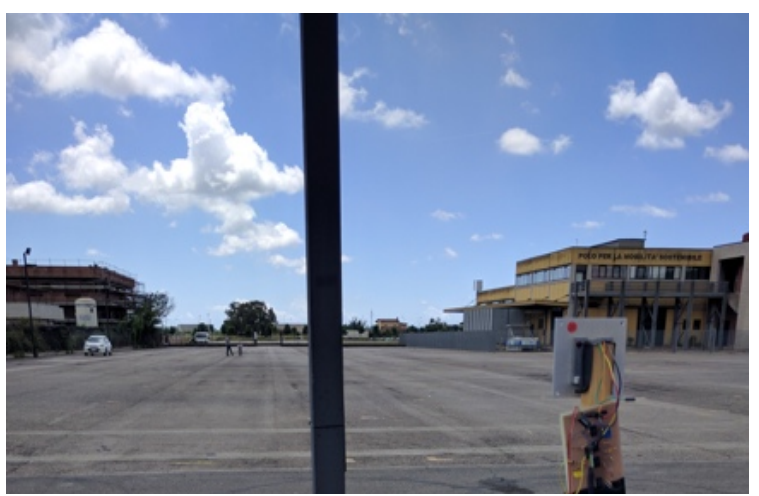

Figure 13. Experimental setup, Pole

As it is shown in Figure 14 (a) at $5 \mathrm{~m}$ distance, pointing the sensors straight to the pole, the obstacle is correctly detected by all sensors, so the Kalman filter gives more trust to the LiDAR which has the smaller measurement noise.

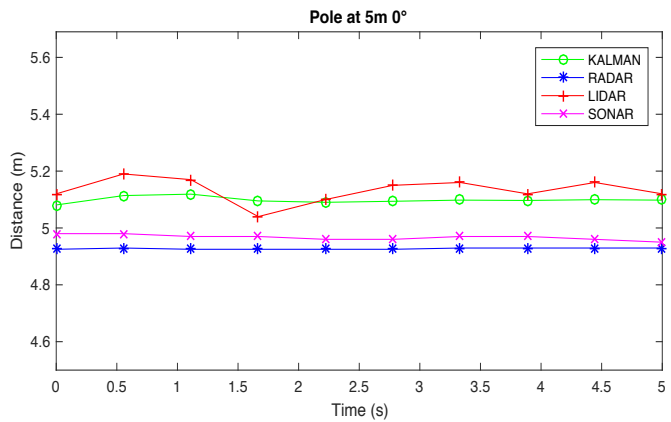

(a)

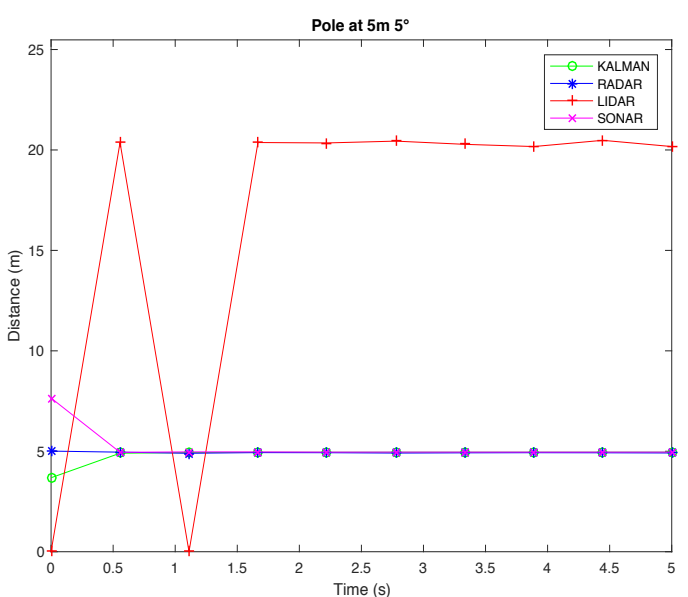

(b)

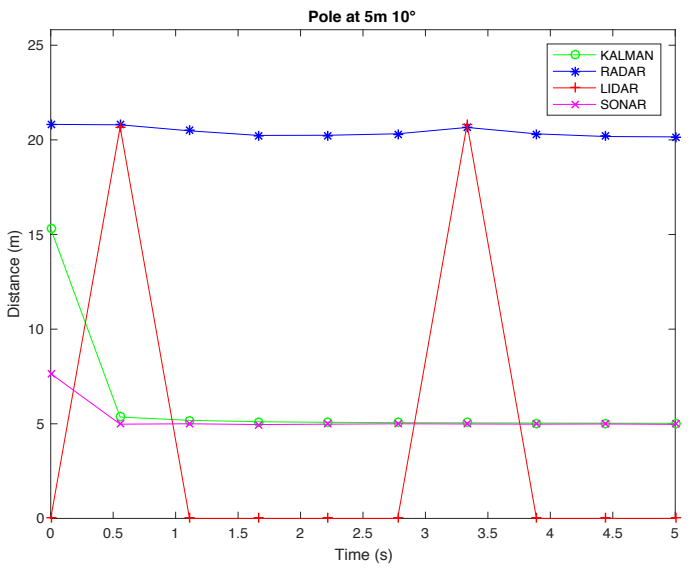

(c)

Figure 14 Pole at $5 \mathrm{~m}$ distance, (a) $0^{\circ}$, (b) $5^{\circ}$, (c) $10^{\circ}$ angle

Figure 14 (b) is taken again at $5 \mathrm{~m}$, but slightly tilting the sensors by few degrees. The LiDAR is no more pointing correctly to the pole thus measuring the background scene, while SONAR and RADAR correctly detect the pole. The Kalman filter output 
follows the latter two sensors. Tilting the sensors more, like in (c), causes the RADAR to lose the target that gets detected just by the SONAR.

Another test has been done by approximately pointing straight to the pole at $15 \mathrm{~m}$ distance (Figure 15). The obstacle is detected just by the RADAR sensor and the Kalman filter converges slightly more slowly.

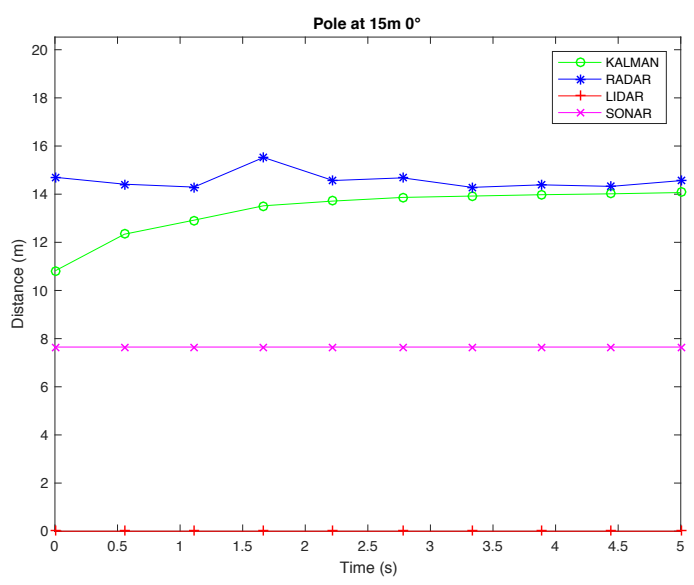

Figure 15 Pole at $15 \mathrm{~m}$ distance, $0^{\circ}$ Angle

4.2.4 Metal Net: A challenging obstacle is a Metal Net (Figure 16), this kind of target is difficult to be detected using a LiDAR or a SONAR due to its form factor while the RADAR easily detects it.

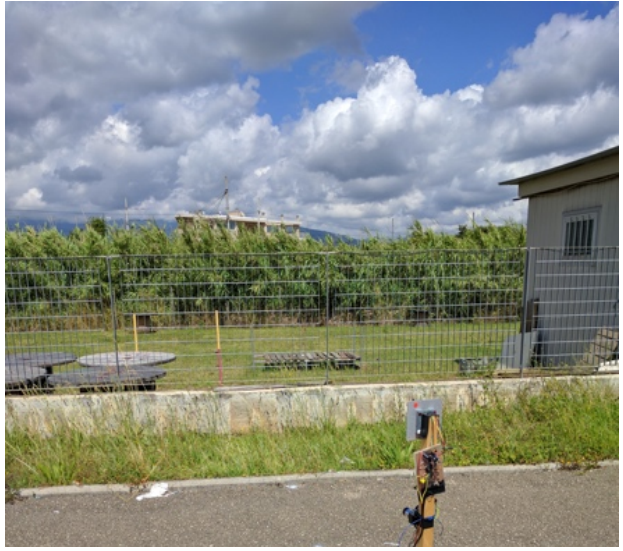

Figure 16. Metal Net, experimental setup

Figure 17 shows the target detection at three different distances, (a) is taken at $3 \mathrm{~m}$ distance, where RADAR and SONAR correctly detect the target, LiDAR is yet failing to give correct measurements. At $5 \mathrm{~m}$ (b) even the SONAR fails to correctly detect the distance. The behavior is like the previous one even increasing the distance from the target as shown in (c).

This case study additionally, demonstrates the importance of a RADAR in detection of sparse metal obstacles like nets and wires.

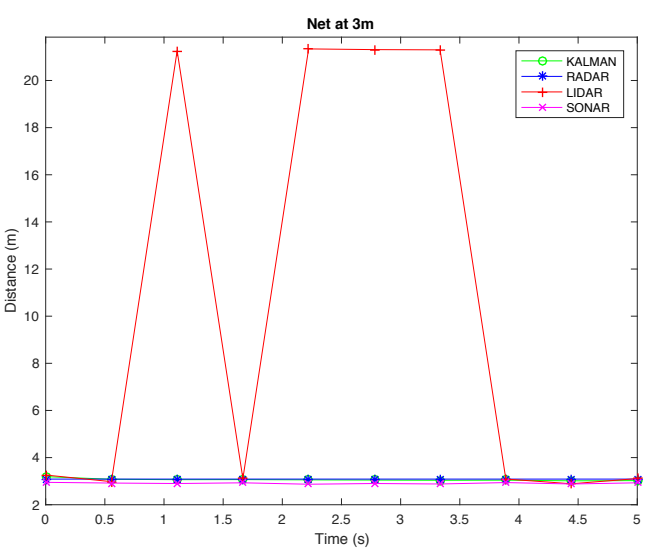

(a)

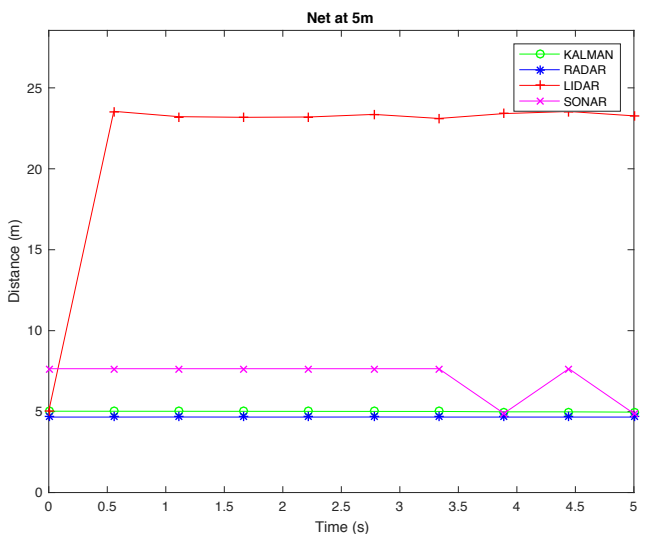

(b)

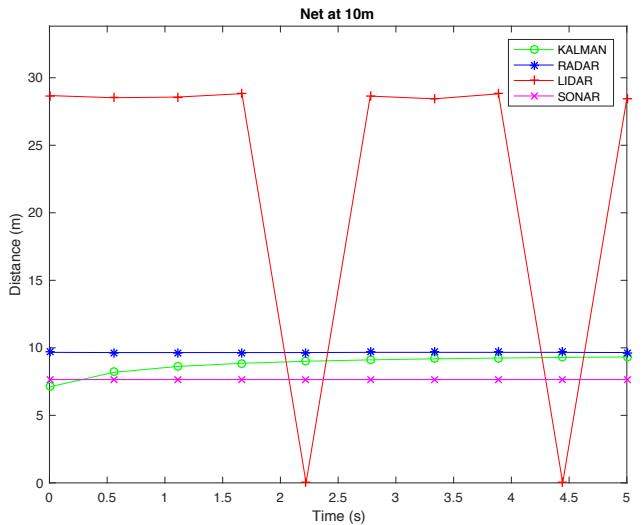

(c)

Figure 17 Metal Net at (a) 3m, (b) 5m, (c) 10m distance.

4.2.5 Person: Another interesting case study is the detection of persons. Figure 18 shows the detection of a person. At $5 \mathrm{~m}$ distance (a) the worst sensor is the SONAR that fails to detect the human as an obstacle. However, LiDAR and RADAR behave correctly and the system detects the person successfully. Going 
further at $10 \mathrm{~m}$ distance as shown in (b), the RADAR too sometimes doesn't see the obstacle but the overall system continues to correctly detect it.

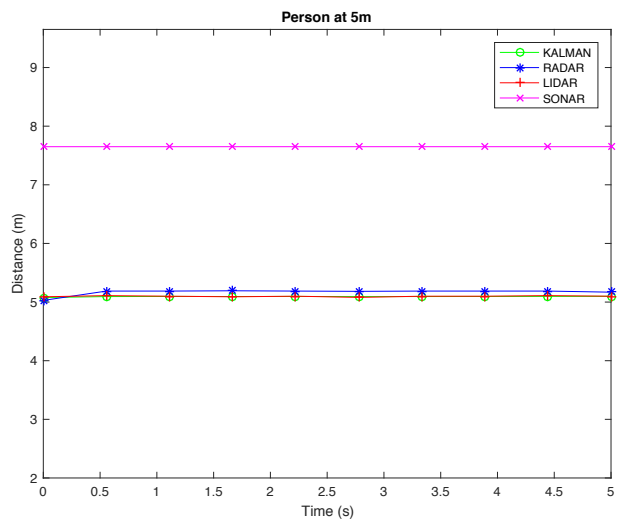

(a)

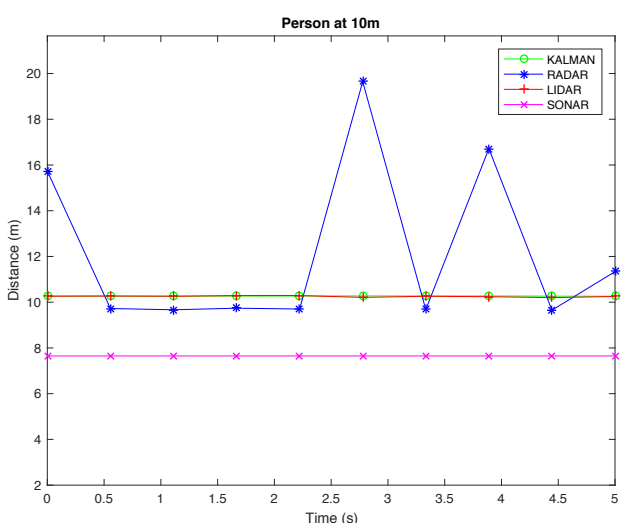

(b)

Figure 18. Person at (a) 5m, (b) $10 \mathrm{~m}$ distance

4.2.6 Vegetation: Vegetation (Figure 19) can be a tough obstacle to detect due to the heterogeneity of its form factor.

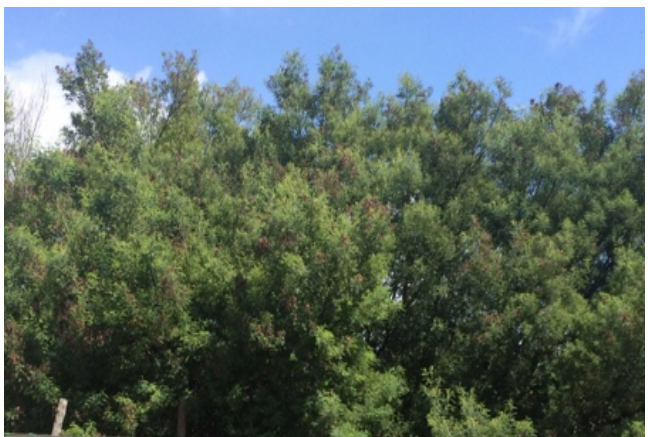

Figure 19. Vegetation obstacle

As can be seen in Figure 20 at $3 \mathrm{~m}$ (a) only the Ultrasonic sensor is easily deceived by this obstacle, while the LiDAR and RADAR behave quite well. Going further with the distance, (b) and (c) show that the best behavior here is the one of LiDAR.

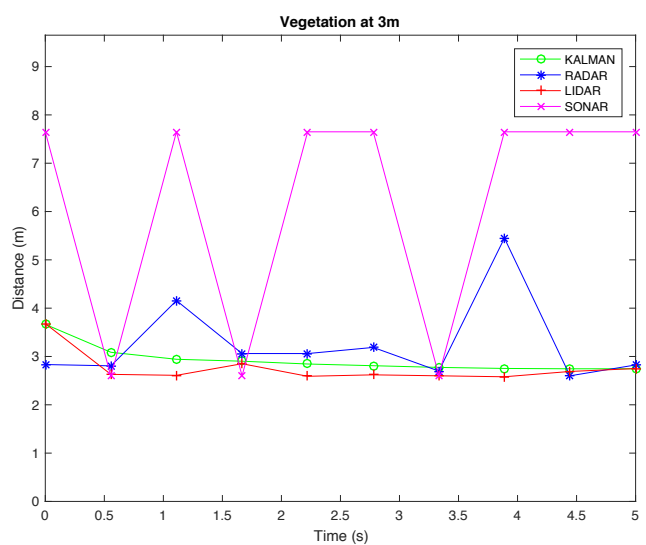

(a)

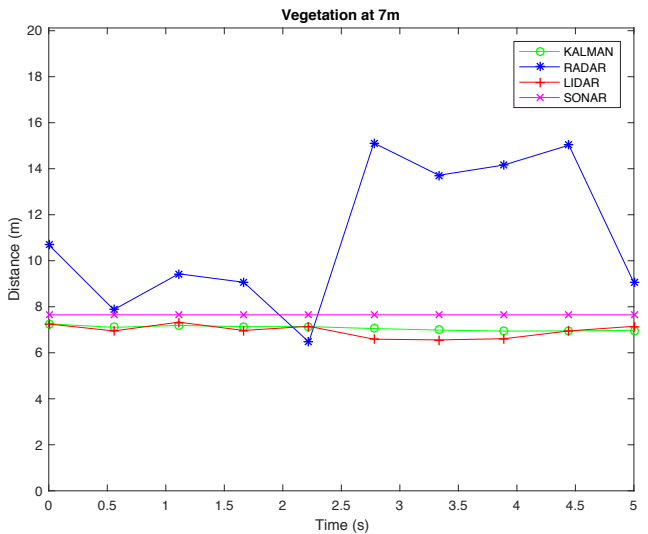

(b)

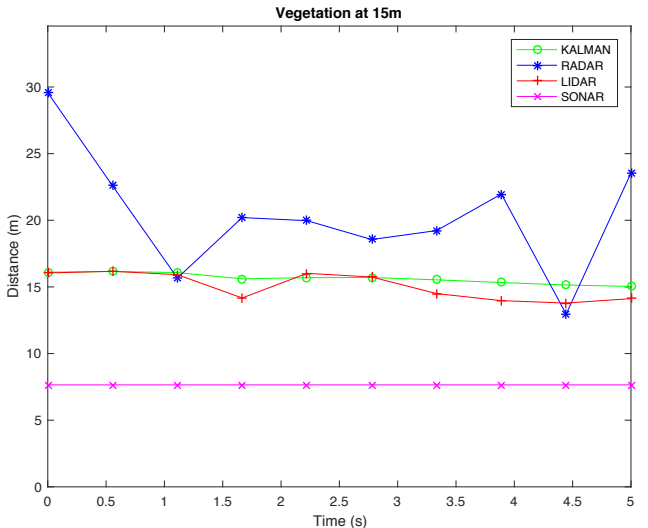

(c)

Figure 20. Vegetation at (a) $3 \mathrm{~m}$, (b) $7 \mathrm{~m}$, (c) $15 \mathrm{~m}$ distance 


\section{CONCLUSIONS}

The multi-sensor approach to obstacle detection using Kalman filter gives promising results: the three sensors complementary capabilities are exploited by the filter, giving an accurate estimation of obstacle distance.

Moreover, the data coming from FMCW RADAR itself are very interesting. In fact, such technology behaves particularly well in all those situations where optical systems fail. However, often practical implementations of obstacle detection systems for UAVs lack this kind of technology because RADAR systems are typically bulky and power thirsty. We have demonstrated that miniaturization of this technology has made possible the integration even on UAVs. For instance, the setup shown in this paper has a power consumption of approximately $2 \mathrm{~W}$ and a weight of less than $0,2 \mathrm{Kg}$.

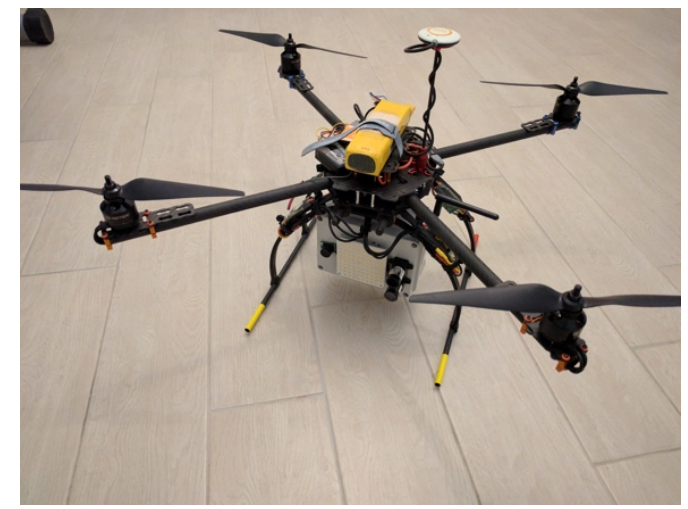

Figure 21 Experimental prototype mounted under a UAV

The next step now is testing this system on board a UAV (Figure 21) to get feedback in real scenarios.

\section{REFERENCES}

Burri M., Oleynikova H., Achtelik M.W., Siegwart R. "Real-time visual-inertial mapping, re-localization and planning onboard MAVs in unknown environments", 2015 IEEE/RSJ Int. Conf. Intelligent Robots and Systems (IROS), DOI 10.1109/IROS.2015.7353622

DJI Mavic Pro, http://www.dji.com/mavic/info\#specs

Fallavollita P., Esposito S., Balsi M., "Perception and decision systems for autonomous UAV flight", in Di Giamberardino P. et al., "Computational Modelling of Objects Represented in Images III Fundamentals, Methods and Applications", CRC Press, 2012 - ISBN 9780415621342

Fasano G., Accardo D., Tirri A.E., Moccia A. "Experimental Analysis of Onboard Non-Cooperative Sense and Avoid Solutions Based on Radar, Optical Sensors, and Data Fusion”, IEEE A\&E Systems Magazine, 2016, DOI 10.1109/MAES.2016.150164

Gageik N., Benz P., Montenegro S., "Obstacle Detection and Collision Avoidance for a UAV With Complementary Low-Cost Sensors", IEEE Access 3, 599-609 (2015), DOI 10.1109/ACCESS.2015.2432455
Gohl P. et al., "Omnidirectional Visual Obstacle Detection using Embedded FPGA," Int. Conf. Intelligent Robots and System (IROS), 2015, DOI: 10.1109/IROS.2015.7353931

Hollinger J., Kutscher B., Closeb R., "Fusion of Lidar and Radar for detection of partially obscured objects", in Karlsen R.E. et al. (ed.) "Unmanned Systems Technology XVII “, Proc. of SPIE VoI. 9468, DOI 10.1117/12.2177050

Innosent GmbH, "Datenblatt_IVS-167_V3.1.pdf," 2013 http://www.innosent.de/fileadmin/media/dokumente/DATASH EETS_2016/Datenblatt_IVS-167_V3.1.pdf

Kanellakis C., Nikolakopoulos G. , "Survey on Computer Vision for UAVs: Current Developments and Trends", J Intell Robot Syst (2017), DOI 10.1007/s10846-017-0483-z

Khaleghi B., Khamis A., Karray F.O., Razavi S.N., "Multisensor data fusion: A review of the state-of-the-art", Information Fusion 14 (2013) 28-44

MaxBotix, "XL-MaxSonar-EZ_Datasheet.pdf" http://www.maxbotix.com/documents/XL-MaxSonarEZ_Datasheet.pdf

Nieuwenhuisen M. et al., "Multimodal Obstacle Detection and Collision Avoidance for Micro Aerial Vehicles, 2013 Eur. Conf. on Mobile Robots (ECMR), DOI: 10.1109/ECMR.2013.6698812

PulsedLight3D, "LIDAR-Lite-v1-docs.pdf," 2015 https://github.com/PulsedLight3D/LIDAR-LiteDocumentation/tree/master/Docs

Sabatini R., Ramasamy S., Gardi A., Rodriguez Salazar L., "Low-cost Sensors Data Fusion for Small Size Unmanned Aerial Vehicles Navigation and Guidance", Int J Unmanned Systems Eng. (2013), 1(3), 16-47

ST Microelectronics, STM32F446xC/E datasheet www.st.com/resource/en/datasheet/stm32f446ze.pdf

Welch G., Bishop G., "An Introduction to the Kalman Filter,"

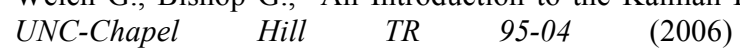
https://www.cs.unc.edu/ welch/media/pdf/kalman_intro.pdf 